\title{
Optimal Mass Diffusion Transfer in Solids Using Heat Transfer Similarities
}

\author{
Samar Jaber ${ }^{1,2}$, Ali Alahmer ${ }^{3,4}$, Gabriel Borowski ${ }^{5 *}$, Sameh Alsaqoor ${ }^{4,6}$ \\ 1 Mechanical Engineering Dept., School of Engineering Technology, Al Hussein Technical University, Jordan \\ 2 Mechanical Engineering Department, American University of Madaba, Madaba, Jordan \\ ${ }^{3}$ Department of Alternative Energy Technology, Faulty of Engineering and Technology, Al-Zaytoonah \\ University, Jordan \\ ${ }^{4}$ Department of Mechanical Engineering, Tafila Technical University, P. O. Box 179, 66110 Tafila, Jordan \\ ${ }^{5}$ Faculty of Environmental Engineering, Lublin University of Technology, ul. Nadbystrzycka 40B, 20-618 Lublin, \\ Poland \\ ${ }^{6}$ Renewable Energy Engineering Department, Faculty of Engineering, Al-Isra University, Amman, Jordan \\ * Corresponding author's e-mail: g.borowski@pollub.pl
}

\begin{abstract}
A new concept of solid-solid mass diffusion fin was introduced in this paper. The authors described the mass diffusion in fluid flows to analyze the transfer process in solids and its applications, especially in metallurgical research. Using the advantage of the similarity between heat transfer and mass diffusion transfer will make this issue more practical. The authors suggested a new approach to determine the maximum mass diffusion transfer between two solids by implementing of the extended surface on each solid, where the optimal mass diffusion between solids is required. Then, meshing the surfaces together in order to increase the transfer efficiency was carried out. A complete analysis of the extended surfaces design (diffusion fins), its efficiency and effectiveness were presented. Moreover, mathematical models of each consideration were constructed. The authors found that the total surface efficiency increased along with the number of mass diffusion fins attached to the base, but its effectiveness did not. The mass diffusion for an extended plate increased the total mass diffusion transfer between two materials.
\end{abstract}

Keywords: diffusion transfer, Fick’s law, diffusion resistance.

\section{INTRODUCTION}

Maximum mass diffusion transfer is the aim of many manufacturing fields. It is defined as the transport of particles by the random vibration of molecules [Crank 1975]. The mass diffusion transfer is commonly used in carburization and many other manufacturing processes and it is governed by the Fick's laws of diffusion. The Fick's law is extensively used as a model for the description of diffusion phenomena, such as heat conduction [Paradisi 2001, Valdes-Parada 2007, Webb and Pruess 2003]. Mejbro [1996] investigated chloride ingression into concrete. It will require solving Fick's second law with time dependent diffusion coefficient and surface concentration.
The author stated that "The heat equation from a mathematical point of view is identical with the simplest equation of diffusion". Furthermore, Zeng et al. [2014] studied the transparency of diffusion of chloride ions into concrete.

Chatterji [1995] discussed the applicability of the Fick's second law to chloride ion migration through Portland cement concrete. The author concluded that there is a fundamental contradiction between the experimental results of the chloride ion migration through the cement and the assumption of a constant diffusion coefficient. Therefore, the diffusivity was considered as a function of time and depth. Lehner [1979] carried out the validity of Fick's law of transient diffusion through a porous medium. He proved mathematically that the 
Table 1. Nomenclature

\begin{tabular}{|cl|cl|}
\hline$c_{1}$ & initial concentration & $D$ & diffusion coefficient (or mass diffusivity) \\
\hline$c_{2}$ & final concentration & $d c / d x$ & concentration gradient \\
\hline$J_{R}$ & mass diffusion rate & $d T / d x$ & temperature gradient \\
\hline$A_{b}$ & area of the base & $k$ & thermal conductivity of the medium \\
\hline$A_{f}$ & area of a single fin & $L$ & fin Length \\
\hline$A_{t}$ & total area for both fins and base & $N$ & number of fins \\
\hline$J_{T}$ & total mass diffusion rate & $P$ & perimeter \\
\hline$r_{0}$ & inner radius & $R$ & mass diffusion resistance, gas constant \\
\hline$r_{1}$ & outer radius & $T$ & absolute temperature \\
\hline$\eta_{T}$ & overall surface efficiency & $\varepsilon$ & effectiveness of a fin \\
\hline$\Delta x$ & thickness of the wall & $\eta$ & efficiency of a fin \\
\hline$A$ & cross-sectional area of the wall &
\end{tabular}

diffusion equation is restricted by the requirements of having quasi-steady diffusion on a pore scale. Moreover, he used the reciprocal theorem in applying the Fick's law on macro level. In turn, Milliigen et al. [2005] investigated the applicability of the Fick's law in non-homogenous material and he concluded that by choosing the appropriate level of approximation for the particle flux, the difficulty of interpreting the Fick's law is negligible.

The effect of mechanical vibrations on diffusion process for a model consists of a gas diffused into a metal (hydrogen in steel) was investigated by Nowacki [1976]. The heat effect on the process of diffusion was neglected to simplify the solution. He eliminated the thermo-diffusion and heat conduction differential equations and examined the displacement and the chemical potential by means of two methods; the elastic potential and basic energy methods.

This manuscript develops a new approach to determine a maximum mass diffusion transfer between two solids by implementing of the extended surface on each solid and use the advantage of the similarity between heat transfer and mass diffusion transfer.

\section{DIFFUSION MASS TRANSFER}

On the basis of the previous and current authors' knowledge, the similarities between heat transfer and mass diffusion transfer are clear in the case of conduction heat transfer and mass diffusion transfer in solids. From this idea, the concept of mass diffusion resistance was introduced. Since the governing equation for conduction heat transfer (Fourier's law) is identical to the mass diffusion transfer equation in solids (Fick's first law) then the behavior of both (heat transfer and mass diffusion) is predicted to be identical.

$$
\frac{\partial}{\partial x}\left(k \frac{\partial T}{\partial x}\right)=0 \text { Fourier's }^{\prime} \text { Law }
$$

$$
\frac{\partial}{\partial x}\left(D \frac{\partial c}{\partial x}\right)=0 F i c k^{\prime} \text { sLaw }
$$

where: $k$ is the thermal conductivity of the medium,

$d T / d x$ is temperature gradient,

$D$ is a diffusion coefficient (or mass diffusivity), and

$d c / d x$ is concentration gradient.

From this principle, the expanding heat transfer applications based on the Fourier's law of conduction to mass diffusion transfer equations will consequentially be valid. If the thermal resistance concept [Fried 1969] is used in diffusion transfer then:

$$
R=\frac{\left|c_{1}-c_{2}\right|}{J_{R}}
$$

where: $R$ is the mass diffusion resistance,

$c_{1}$ is the initial concentration,

$c_{2}$ is the final concentration and

$J_{R}$ is the mass diffusion rate.

Similarly, for a series connection, the equivalent resistance is expected to be the summation of the resistances of all materials along the path of mass transfer, while in the parallel connection the equivalent diffusion resistance is the sum of the reciprocals of the diffusion resistances of the materials perpendicular to the path of diffusion transfer. 
Using the mass diffusion resistance equation depends on the rate of mass diffusion $\left(J_{R}\right)$. However, $\left(J_{R}\right)$ is found by $J_{R}=D A \frac{d c}{d x}$. Noting that the slope $\left(\frac{d c}{d x}\right)$ is depending on the profile of the concentration. Thus, for a linear profile (plane wall): $J_{R}=\frac{D}{\Delta x} A \Delta C$ and $J_{R}=\frac{2 \pi L D}{\ln \left(\frac{r_{1}}{r_{0}}\right)} * \Delta C$ for
the cylindrical case.

By applying the mass diffusion resistance concept on the mentioned cases (plane wall and a cylinder):

Plane Wall Resistance:

$$
R=\frac{\Delta C}{J_{R}}=\frac{\Delta C}{\frac{D}{\Delta x} A \Delta C}=\frac{\Delta x}{D A}
$$

where: $D$ is the mass diffusivity of the material, $\Delta \chi$ is the thickness of the wall and $A$ is the cross-sectional area of the wall.

Cylinder resistance:

$$
R=\frac{\Delta C}{J_{R}}=\frac{\Delta C}{\frac{2 \pi L D}{\ln \left(\frac{r_{1}}{r_{0}}\right)} * \Delta C}=\frac{\ln \left(\frac{r_{1}}{r_{0}}\right)}{2 \pi L D}
$$

where: $r_{1}$ and $r_{0}$ are the outer and inner radii, respectively.

\section{EXTENDED SURFACES FOR MASS DIFFUSION TRANSFER}

Extended surfaces are extension solid surfaces that are connected to the boundaries of the base of the solid to enhance the base solid ability for heat or mass transfer [Cengel and Ghajar 2014]. The main goal here is to ensure the maximum mass diffusion transfer between material A and B as depicted in Figure 1.

Unlike fins in heat transfer, the introduced mass diffusion fin here has a mass transfer from a solid to a solid, rather than from a solid to a fluid. Therefore, the similarity of solutions of fins will occur.

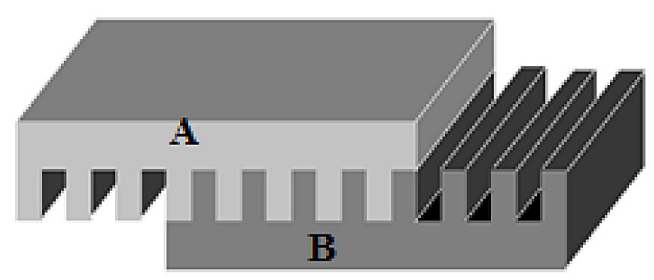

Figure 1. Different mass diffusion fins, material $\mathrm{A}$ - donor/receiver; material B - receiver/donor

\section{Mathematical derivation of the general mass diffusion transfer in a fin}

In order to find a governing equation to describe the mass diffusion transfer in fins, a steadystate mass balance was done. A control volume for a non-uniform section fin was chosen as displayed in Figure 2.

Examining Figure 2 shows that:

$$
J_{x}=J_{x+d x}+d J_{P}
$$

while:

$$
\begin{aligned}
& J_{x}=D_{1} A_{c} \frac{d c}{d x} J_{x+d x}=J_{x}+\frac{d J_{x}}{d x} d x \\
& d J_{P}=D_{2} d A_{s}\left(C-C_{\infty}\right)
\end{aligned}
$$

where: $D_{1}=D_{A^{\prime}} D_{2}=D_{E^{*}}$

$D_{E}$ is a fin that will mesh with this fin perfectly as shown in Figure 2.

Substituting back to equation (6) yields:

$$
\begin{aligned}
D_{1} A_{c} \frac{d c}{d x} & -\left(D_{1} A_{c} \frac{d c}{d x}+\frac{d}{d x}\left(D_{1} A_{c} \frac{d c}{d x}\right) d x\right)- \\
& -D_{2} d A_{s}\left(C-C_{\infty}\right)=0
\end{aligned}
$$

Canceling terms yields:

$$
\frac{d}{d x}\left(D_{1} A_{c} \frac{d c}{d x}\right) d x-D_{2} d A_{s}\left(C-C_{\infty}\right)=0
$$

Dividing by $\mathrm{D}_{1} \mathrm{~d}_{\gamma}$ :

$$
\frac{d}{d x}\left(D_{1} A_{c} \frac{d c}{d x}\right)-\frac{D_{2}}{D_{1}} \frac{d A_{s}}{d x}\left(C-C_{\infty}\right)=0
$$

Taking the derivatives in the equation yields:

$$
A_{c} \frac{d^{2} c}{d x^{2}}+\frac{d c}{d x} \frac{d A_{c}}{d x}-\left(\frac{D_{2}}{D_{1}} \frac{d A_{s}}{d x}\left(C-C_{\infty}\right)\right)=0
$$

Dividing by $\mathrm{A}_{\mathrm{c}}$ :

$$
\frac{d^{2} c}{d x^{2}}+\left(\frac{1}{A_{c}} \frac{d c}{d x} \frac{d A_{c}}{d x}\right)-\left(\frac{1}{A_{c}} \frac{D_{2}}{D_{1}} \frac{d A_{s}}{d x}\left(C-C_{\infty}\right)\right)=0
$$

Equation (12) governs the mass diffusion transfer for uniform and non-uniform cross sectional area fins. 

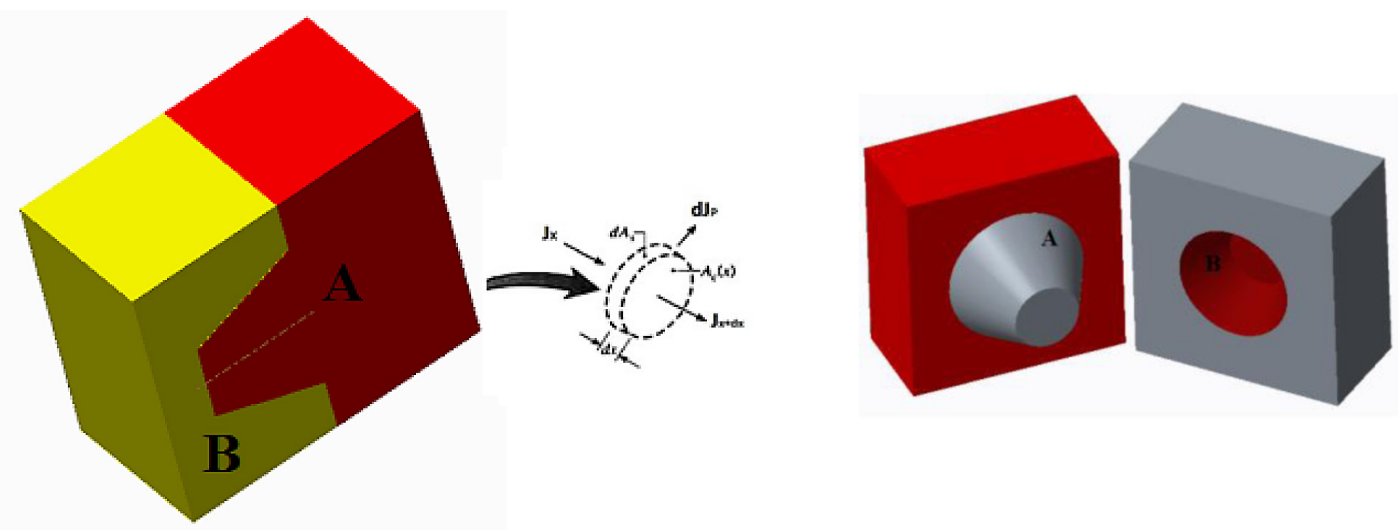

Figure 2. Control volume a non-uniform section fin

\section{Extended plate with uniform cross-sectional area}

For a constant cross sectional area [Harper and Brown 1922], the following terms are applied back to equation (12):

$$
\frac{d A_{c}}{d x}=0, A_{s}=P x
$$

where: $P$ is the perimeter.

The $P \chi$ term is the function that will govern the area.

Plugging those assumptions back to equation (12) yields [Incropera et al. 2011]:

$$
\begin{gathered}
\frac{d^{2} c}{d x^{2}}-\frac{P D_{2}}{A_{c} D_{1}}\left(C-C_{\infty}\right)=0 \\
\text { let } \alpha=c-c_{\infty} \wedge \omega^{2}=\frac{P D_{2}}{A_{c} D_{1}} \text { then }: \\
\frac{d^{2} \alpha}{d x^{2}}-\omega^{2} \alpha=0
\end{gathered}
$$

Equation (12) is a 2nd order, homogeneous and ordinary differential equation [Zill 2016]. Since we have only two constants, thus, only two boundary conditions are needed. The first one is at $\chi=0, \alpha=\alpha_{\text {base }}$. While the second boundary condition depends on what assumptions were made. The four common assumptions are [Holman 2009, Callister 2013]:

1. Very Long Fin.

2. Finite length with an insulated tip.

3. Finite length with diffusion transfer at the tip.

4. At a prescribed temperature.

\section{Case 1: Very Long Fin}

Applying the first boundary condition on the general solution (G.S) yields:

$$
\propto_{b}=C_{1}+C_{2}
$$

The second boundary condition is:

$$
x \rightarrow \infty, c \rightarrow c_{\infty} \wedge \alpha \rightarrow 0
$$

Plugging it back to the G.S, produces;

$$
\frac{\alpha}{\alpha_{b}}=e^{-\omega x}
$$

Equation (18) is referred to as the profile of the transfer process, further simplification yields:

$$
\begin{aligned}
& \frac{c-c_{\infty}}{c_{b}-c_{\infty}}=e^{-\omega x} \\
& c-c_{\infty}=\left(c_{b}-c_{\infty}\right) * e^{-\omega x} \\
& \frac{d c}{d x}=-\omega\left(c_{b}-c_{\infty}\right) e^{-\omega x}
\end{aligned}
$$

$$
\begin{aligned}
& \text { When } \rightarrow 0 \text { : } \\
& \qquad \begin{array}{l}
\quad \therefore=-D_{1} A *\left(-\omega\left(c_{b}-c_{\infty}\right)\right) \\
J=D_{1} A \omega\left(c_{b}-c_{\infty}\right)
\end{array}
\end{aligned}
$$

or:

$$
J=\sqrt{D_{2} D_{1} P A} \alpha_{b}
$$

Equation (21) gives the total amount of mass diffusion transfer in a fin with infinite length (the actual length is infinite relative to the base). In other words, a finite length can be considered as infinitely far away from the base [Incropera et al. 2011]. 


\section{Case 2: Finite length with an insulated tip}

The first boundary condition is:

$$
\propto_{b}=C_{1}+C_{2}
$$

The second boundary condition is:

$$
\text { atx }=L,\left(\frac{d c}{d x}\right)_{x=L}=0,\left(\frac{d \alpha}{d x}\right)_{x=L}=0
$$

Plugging the two boundary conditions into the G.S gives;

$$
\frac{\alpha}{\alpha_{b}}=\frac{\cosh [\omega *(L-x)]}{\cosh (\omega L)}
$$

Equation 24 is the mass diffusion transfer profile along the fin with finite length and insulated tip.

Taking the derivative of the profile:

$$
\frac{d \alpha}{d x}=\frac{d}{d x}\left(\alpha_{b}\left(\frac{\cosh [\omega *(L-x)]}{\cosh (\omega L)}\right)\right)
$$

Simplifying and plugging it back to $J=-D_{1} A \frac{d \alpha}{d x}$ yields:

$$
J=\sqrt{D_{2} D_{1} P A} \alpha_{b} \tanh (\omega L)
$$

Similarly, this is the mass diffusion transfer equation for this case.

\section{Case 3: Finite length with diffusion transfer at the tip}

The first boundary condition is:

$$
\propto_{b}=C_{1}+C_{2}
$$

The second boundary condition is at $\mathrm{x}=\mathrm{L}$. The solution for finite length with diffusion transfer at the tip is:

$$
\frac{\alpha}{\alpha_{b}}=\frac{\cosh \omega(L-x)+\left(\frac{1}{\omega} \frac{D_{2}}{D_{1}}\right) \sinh \omega(L-x)}{\cosh \omega L+\left(\frac{1}{\omega} \frac{D_{2}}{D_{1}}\right) \sinh \omega L} \ldots(11)
$$

Where equation (27) is the mass diffusion profile. Taking the derivative and plugging back to $J=-D_{1} A \frac{d \alpha}{d x}$

$$
J=\sqrt{D_{2} D_{1} P A} \alpha_{b} \frac{\sinh \omega L+\left(\frac{D_{2}}{\omega D_{1}}\right) \cosh \omega L}{\cosh \omega L+\left(\frac{D_{2}}{\omega D_{1}}\right) \sinh \omega L}
$$

Equation (29) is the mass diffusion transfer equation for a fin with finite length and mass diffusion transfer at the tip. The transfer equation for case (4) can be similarly constructed.

For the ratio $\frac{D_{2}}{D_{1}}[19]$, where $\mathrm{D}_{1}=\mathrm{D}_{\mathrm{A}}, \mathrm{D}_{2}=\mathrm{D}_{\mathrm{E}}$ :

$$
D=D_{0} * e^{\frac{-Q}{R T}}
$$

where: $D_{0}$ is a temperature-independent Preexponential,

$Q$ is the activation energy for diffusion,

$R$ is the gas constant and

$T$ is the absolute temperature. Using this formula:

$$
\frac{D_{2}}{D_{1}}=\frac{D_{0} * e^{\frac{-Q}{R T_{2}}}}{D_{0} * e^{\frac{-Q}{R T_{1}}}}=e^{\frac{-Q}{R T_{2}}} * e^{\frac{Q}{R T_{1}}}=e^{\frac{Q}{R}\left(\frac{1}{T_{1}}-\frac{1}{T_{2}}\right)}
$$

\section{Performance of mass diffusion fins}

The purpose of fins is to increase the mass diffusion transfer for the system. The effectiveness $(\varepsilon)$ definition is the ratio between the mass diffusion rate and the mass diffusion rate that would exist without the fin. Thus:

$$
\varepsilon=\frac{J_{\text {fin }}}{D_{2} A_{c} \alpha_{b}}
$$

where: $J_{\text {fin }}$ is the amount of mass diffusion transfer in the fin, $\alpha b=c b-\infty$.

The effectiveness in terms of mass diffusion resistances:

$$
\varepsilon=\frac{R_{b}}{R_{\text {fin }}}
$$

While the efficiency of a fin $(\eta)$ is defined as mass diffusion in the fin $\left(\mathrm{J}_{\text {fin }}\right)$ divided by the maximum mass diffusion rate $\left(\mathrm{J}_{\text {Max }}\right)$, the mass diffusion that would exist if the entire fin is at the base concentration)[20].

$$
\eta=\frac{J_{\text {fin }}}{J_{\operatorname{Max}}}=\frac{J_{\text {fin }}}{D_{2} A_{f} \alpha_{b}} \ldots
$$

\section{Overall surface efficiency for mass diffusion fins}

Usually, a single fin is not sufficient to the overall desired transfer, in practical applications an array of fins is used and finding the efficiency of every single fin individually is tedious. 
Therefore, the overall efficiency is considered as follows:

$$
\eta_{T}=\frac{J_{T}}{J_{M a x}}=\frac{J_{T}}{D_{2} A_{t} \alpha_{b}}
$$

where: $\eta_{r}$ is the overall surface efficiency,

$J_{r}$ is the total mass diffusion rate from the surface and $A_{t}$ is the total area for both fins and base.

The mathematical expression for $A_{t}$ is [Incropera et al. 2011]:

$$
A_{t}=N A_{f}+A_{b} \ldots
$$

where: $N$ is the number of fins attached to the base,

$A_{f}$ is the area of a single fin and

$A_{b}$ is the area of the base.

Applying the same principle for the mass diffusion transfer:

$$
\begin{aligned}
& J_{T}=J_{\text {Fins }}+J_{\text {Base }} \\
& J_{T}=N \eta_{f} D_{2} A_{f} \alpha_{b}+D_{2} A_{b} \alpha_{b}
\end{aligned}
$$

where: $\eta_{f}$ is the efficiency of a single fin.

$$
\text { Knowing } \mathrm{Ab}=\mathrm{At}-\mathrm{NA}_{\mathrm{f}} \text { Thus: }
$$

$$
J_{T}=D_{2} A_{t} \alpha_{b}\left(1-\frac{N A_{f}}{A_{t}}\left(1-\eta_{f}\right)\right)
$$

Substituting this back to the main efficiency formula (equation 35):

$$
\eta_{T}=1-\frac{N A_{f}}{A_{t}}\left(1-\eta_{f}\right)
$$

\section{CONCLUSIONS}

The similarity between heat transfer and mass diffusion transfer is very clear with both diffusion resistance and extended surface concepts. The new concept from the similarities in the governing equations introduces an application area (mass diffusion fins) that can be used for the manufacturing purposes. It was shown that if the number of mass diffusion fins attached to the base is increased, the total surface efficiency increase as well, but not its effectiveness. The authors concluded that the mass diffusion for an extended plate increases the total mass diffusion transfer between two materials. Hence, it is more efficient to design the mass diffusion transfer processes based on the design criteria mentioned in the work.

\section{REFERENCES}

1. Callister W., 2013. Materials science and engineering: An introduction. John Wiley \& Sons.

2. Cengel Y. and Ghajar A., 2014. Heat and mass transfer: fundamentals and applications. April 4, McGraw-Hill Education.

3. Chatterji S., 1995. On the applicability of Fick's second law to chloride ion migration through portland cement concrete. Cement and Concrete Research, 25(2), 299-303.

4. Crank J., 1975. The mathematics of diffusion. Clarendon Press.

5. Fried E., 1969. Thermal conduction contribution to heat transfer at contacts. Academic Press London,

6. Incropera F.P., Dewitt D., Bergman T.L., Lavine S.A., 2011. Principles of heat and mass transfer. John Wiley \& Sons.

7. Harper R. and W. Brown, 1922. Mathematical equations for heat conduction in the fins of aircooled engines. NACA Report No. 158,

8. Holman J.P., 2009. Heat transfer. McGraw-Hill Education.

9. Lehner F.K., 1979. On the validity of Fick's law for transient diffusion through a porous medium. Chemical Engineering Science, 34(6), 821-825.

10. Mejlbro L., 1996. The complete solution of Fick's second law of diffusion with time-dependent diffusion coefficient and surface concentration. Technical University of Denmark.

11. Milligen B.P.V., et al., 2005. On the applicability of Fick's law to diffusion in inhomogeneous systems. European Journal of Physics, 26(5), 913-925.

12. Nowacki W., 1976. Dynamic problems of diffusion in solids. Engineering Fracture Mechanics, 8(1), 261-266.

13. Paradisi P. et al., 2001. The fractional Fick's law for non-local transport processes. Physica A: Statistical Mechanics and its Applications, 293(1), 130-142.

14. Valdes-Parada F.J., Ochoa-Tapia J.A., AlvarezRamirez J., 2007. Effective medium equations for fractional Fick's law in porous media. Physica A: Statistical Mechanics and its Applications, 373(Supplement C), 339-353.

15. Webb S.W. and Pruess K., 2003. The use of Fick's law for modeling trace gas diffusion in porous media. Transport in Porous Media, 51(3), 327-341.

16. Zeng L. et al., 2014. Experimental measure mass diffusion transparency. Solid State Communications, 186 (Supplement C), 23-27.

17. Zill D.G., 2016. Advanced engineering mathematics. Jones \& Bartlett Learning. 